\title{
Cluster production within transport theory
}

\author{
AKIRA ONO
}

Department of Physics, Tohoku University, Sendai 980-8578, Japan

\begin{abstract}
Clusters are quite important at various situations in heavy-ion collisions and in excited low-density nuclear matter. Antisymmetrized molecular dynamics has been improved to take into account the correlations to form light clusters such as deuterons and $\alpha$ particles. The present status of this approach for the description of multifragmentation is reported. Calculated results demonstrate strong impacts of clusters in various observables including those usually regarded as probes of the density dependence of symmetry energy.
\end{abstract}

\section{Introduction}

Fragments and light clusters are copiously produced in various situations in heavy-ion collisions. For example, in $\mathrm{Xe}+\mathrm{Sn}$ central collisions at 50 $\mathrm{MeV} /$ nucleon, the INDRA data [1] show that only $10 \%$ of total protons in the system are emitted as free protons and all the other protons are bound in light clusters, such as $\alpha$ particles, and in heavier fragment nuclei. Even though the incident energy is raised, the free-proton ratio increases only gradually [2]. A similar feature can be found in the data of Ref. [3] which shows the cluster mass fractions as functions of the excitation energy of the vaporized ${ }^{36} \mathrm{Ar}$ projectile nucleus. The cluster composition in excited low-density nuclear matter can also be an important factor to describe corecollapse supernovae [4]. 
Recently the heavy-ion data from Texas A\&M have been analyzed to extract the cluster composition and other properties of nuclear matter as functions of the evolution of the temperature and the density [5]. Comparison of the extracted equilibrium constants for the cluster composition with various equation-of-state (EOS) models suggests necessity of a cluster suppression mechanism such as medium effects [6]. Nevertheless, in general, the link between heavy-ion collisions and EOS should be carefully checked because of the dynamical nature of collisions.

The property of a cluster in medium is typically calculated with an inmedium Schrödinger equation as in Ref. [7]. A cluster can exist as a bound state, depending on the density of the medium and the momentum of the cluster relative to the medium. Then, in actual systems, many clusters may coexist and change the nuclear matter properties and the collision dynamics.

It is therefore demanded that the transport models should solve the time evolution of collisions by correctly treating cluster correlations. However, transport models have been developed based on the single-nucleon motions, and only few models treat cluster correlations explicitly. In a version of BUU by Danielewicz et al. [8], clusters up to ${ }^{3} \mathrm{H}$ and ${ }^{3} \mathrm{He}$ have been introduced as new particle species and the equations for the distribution functions are coupled by the collision terms representing various reactions of clusters and nucleons. A recent calculation demonstrates that collision dynamics is influenced by the clusters [9]. On the other hand, in the antisymmetrized molecular dynamics (AMD) approach, we have introduced cluster correlations by improving the two-nucleon collision procedure [10]. The aims of the rest of this article are to report the present status of this approach and to demonstrate the importance of clusters in the studies of heavy-ion collisions.

\section{$2 \quad$ AMD with clusters}

In AMD, a many-body state is described by a Slater determinant of Gaussian wave packets. The time evolution of the wave-packet centroids is determined by the motion in the mean field, derived from the time-dependent variational principle, and the stochastic two-nucleon collision process. The latter is a quantum mechanical transition from an AMD state to another AMD state with a transition rate

$$
W_{i \rightarrow f}=\frac{2 \pi}{\hbar}\left|\left\langle\Psi_{f}|V| \Psi_{i}\right\rangle\right|^{2} \delta\left(E_{f}-E_{i}\right) .
$$

The most basic version is described in Ref. [11], where only the states of the colliding nucleons are changed by a two-nucleon collision. 
It has been known that this basic version of AMD does not well reproduce the fragmentation in collisions of heavy nuclei such as $\mathrm{Xe}+\mathrm{Sn}$ central collisions at $50 \mathrm{MeV} /$ nucleon, with serious underproduction of $\alpha$ particles and overproduction of nucleons (see Ref. [10]). It should be noted that the AMD wave function is suitable for describing cluster correlations in the ground state and low-lying excited states of nuclei [12]. The problem is the too small probability that clusterized states are realized during the evolution of reactions. In fact, after a two-nucleon collision, one (or both) of the scattered nucleons may accidentally form a cluster with surrounding nucleon(s). The probability of this kind of cluster formation should be calculated as a transition to a specific quantum state because it does not necessarily agree with the classical phase space to form a cluster.

Therefore, in the new version of AMD with cluster correlations, the transitions to the clusterized final states are explicitly taken into account, by including those states in the set of final states $\left\{\left|\Psi_{f}\right\rangle\right\}$. When two nucleons $N_{1}$ and $N_{2}$ collide, we consider the cluster-forming reaction $N_{1}+B_{1}+N_{2}+B_{2} \rightarrow$ $C_{1}+C_{2}$ in the most general case. Here $B_{1}\left(B_{2}\right)$ is a nucleon or a set of nucleons which is forming a cluster $C_{1}\left(C_{2}\right)$ with the nucleon $N_{1}\left(N_{2}\right)$. The transition rates are still given by Eq. (1). See Ref. [10] for more details. We consider the formation of clusters up to $\alpha$ clusters as $C_{1}$ and $C_{2}$. A cluster is represented by placing the Gaussian wave packets of nucleons at the same phase-space point, so that the overlap probabilities from the matrix element in Eq. (1) are expressed analytically. The interaction $V$ in Eq. (1) is that between $N_{1}$ and $N_{2}$, and therefore we need the two-nucleon matrix elements which are essentially given once we have chosen a parametrizattion of twonucleon collision cross sections which may be modified in medium. For example, in the calculations in the next section, we have chosen a so-called screened cross section [9]

$$
\sigma_{\mathrm{NN}}=\sigma_{0} \tanh \left(\sigma_{\text {free }} / \sigma_{0}\right), \quad \sigma_{0}=y \rho^{-2 / 3}, \quad y=0.85 .
$$

No parameters have been introduced to adjust individual probabilities of different cluster formation reactions, but the overall cluster production probability is suppressed for low momentum-transfer collisions by a factor $1-\exp \left[-\mathbf{q}^{2} /(64 \mathrm{MeV} / c)^{2}\right]$. There are many possible final states $\left\{\left|\Psi_{f}\right\rangle\right\}$ corresponding to different ways to form clusters. The non-orthogonality of these states should be carefully handled [10]. Finally, it should be commented that the formed cluster $\left(C_{1}\right.$ or $\left.C_{2}\right)$ is treated in the following time evolution just as several nucleons which are accidentally at the same point (except for the extension in the next paragraph). A cluster may be broken by the mean field or by a two-nucleon collision between a nucleon in it and 
another nucleon in the system like a process $d+X \rightarrow n+p+X^{\prime}$. It is also possible that, in the final state of this two-nucleon collision, the same cluster is formed again like an elastic process $d+X \rightarrow d+X^{\prime}$. Thus, without introducing many parameters, various kinds of cluster reactions have been introduced.

Many of light nuclei ( $\mathrm{Li}, \mathrm{Be}$ etc.) have only one or a few bound states which may be regarded as bound states of internal clusters. Then the same issue exists about the mismatch of classical phase space and the quantummechanical transition. Therefore, for a better description, inter-cluster correlation is introduced as a stochastic process of binding clusters. The basic idea is to replace the relative momentum between clusters by zero if moderately separated clusters $\left(1<R_{\text {rel }}<5 \mathrm{fm}\right)$ are moving away from each other with a small relative momentum $\left(\mathbf{R}_{\text {rel }} \cdot \mathbf{P}_{\text {rel }}<0\right.$ and $\left.P_{\text {rel }}^{2} / 2 \mu<E_{\text {rel }}^{\max }\right)$. It is allowed that more than two clusters are linked by this condition and to be bound simultaneously. The energy conservation should be achieved by scaling the relative momentum between the center-of-mass of the bound clusters (CC system) and a third particle chosen with a suitable criterion. The numerical results depend on the adopted conditions for this cluster-cluster binding process. In the following calculations, we have chosen $E_{\mathrm{rel}}^{\max }=8$ $\mathrm{MeV}$, but it is reduced by a factor $e^{-\left(A_{\mathrm{CC}} / 35\right)^{2}}$ when many clusters (with total mass number $A_{\mathrm{CC}}$ of the CC system) are to be bound simultaneously. The binding is not considered if $10 \leq A_{\mathrm{CC}} \leq 18$, in order to compensate the issue of the overbinding of the nuclei in this mass region with the adopted effective interaction.

\section{Basic characters of multifragmentation}

In this section, the calculated fragment charge distributions for multifragmentation in some central collisions are shown in comparison with experimental data. All the calculations were performed with the AMD with cluster correlations with the same model parameters as described in the previous section. The Skyrme SLy4 interaction was adopted with the spin-orbit term neglected. The decays of excited fragments at the end of the AMD calculation were calculated by a statistical decay code.

Figure 1 shows the comparison for $\mathrm{Xe}+\mathrm{Sn}$ collisions at 50 and 32 $\mathrm{MeV} /$ nucleon. The light particle multiplicities (multiplied by the particle mass number) are also shown in the inset. The overall distribution of the fragments $(Z \geq 3)$ and the individual multiplicities of light charged particles are all reproduced very well. The deviations in the yields around 

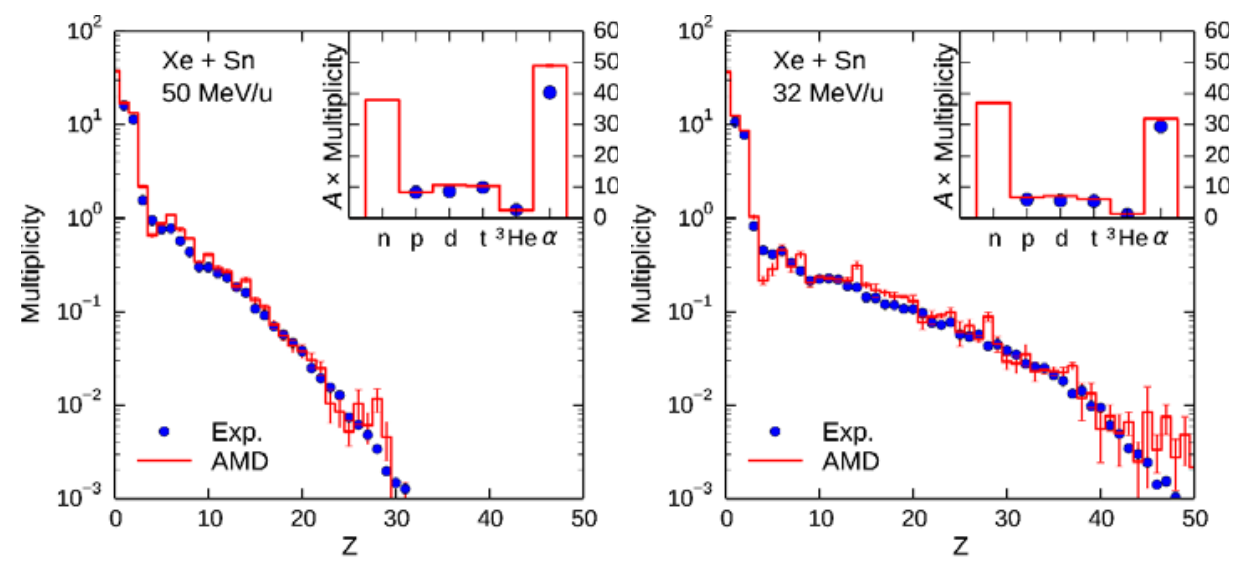

Figure 1: Fragment charge distribution in central Xe + Sn collisions $(0<b<2$ $\mathrm{fm}$ ) at the incident energies of 50 (left) and 35 (right) $\mathrm{MeV} /$ nucleon, calculated by AMD with cluster correlations. The inset shows the multiplicities of light particles multiplied by the mass number. The INDRA experimental data are taken from Ref. [1].

$Z=4$ at both energies have not been well understood. As well as the cluster correlations (to form clusters up to $\alpha$ particles), the cluster-cluster correlations are quite important to reproduce the data at both incident energies simultaneously.

The fragment multiplicities were calculated by the same model for violent collisions of ${ }^{40} \mathrm{Ca}+{ }^{40} \mathrm{Ca}$ at $35 \mathrm{MeV} /$ nucleon (left part of Fig. 2) and central collisions of $\mathrm{Au}+\mathrm{Au}$ at $250 \mathrm{MeV} /$ nucleon (right part of Fig. 2). The reproduction of data is almost satisfactory if we consider the wide difference of the system sizes and the incident energies. The $\alpha$ particles in $\mathrm{Ca}+\mathrm{Ca}$ at $35 \mathrm{MeV} /$ nucleon are underproduced in contrast to the overproduction in $\mathrm{Xe}+\mathrm{Sn}$ at $50 \mathrm{MeV} /$ nucleon. However, the reproductions of these two systems are much more consistent than with any other previous versions of AMD. The yield of relatively heavy fragments in the $\mathrm{Au}+\mathrm{Au}$ collision may depend on the details of the cluster-cluster binding process.

The yields of clusters and fragments in these various reaction systems represent the essential information on how many-body spatial correlations develop in expanding systems with various expansion speeds and/or thermal excitations. A consistent description of this feature is an important milestone to enable precise studies of other features, as in the following sections. 

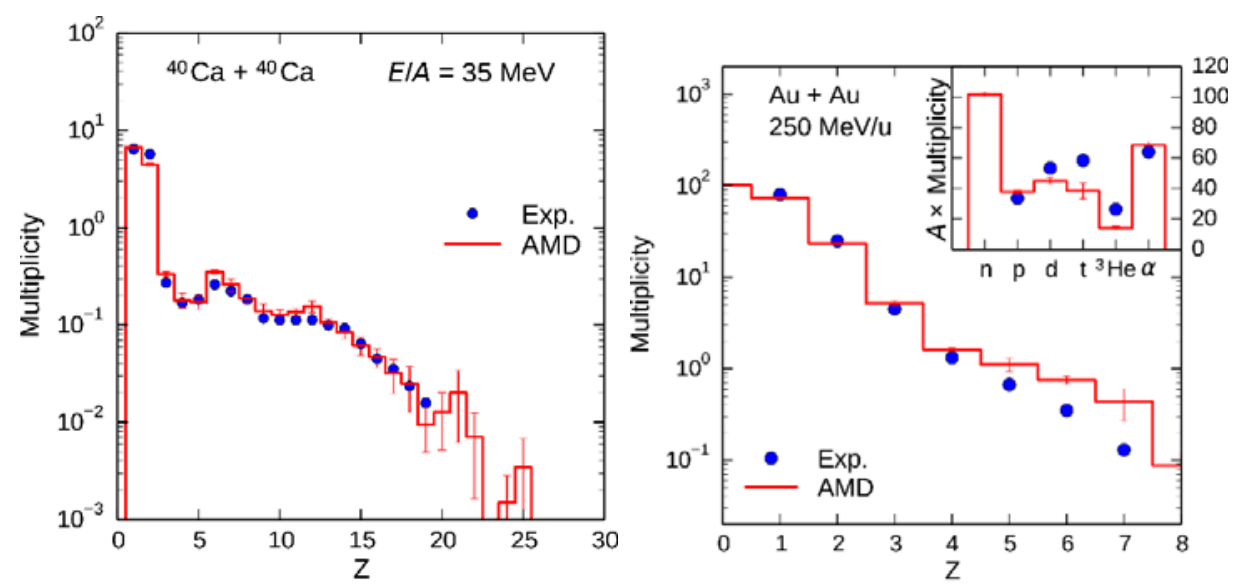

Figure 2: Left: Fragment charge distribution in violent events in ${ }^{40} \mathrm{Ca}+{ }^{40} \mathrm{Ca}$ collisions at $35 \mathrm{MeV} /$ nucleon. A filter for the experimental setup has been applied to the calculated events, and the violent events are selected by the same condition as for the experimental data [13]. Right: Fragment charge distribution in central $\mathrm{Au}+\mathrm{Au}$ collisions at $250 \mathrm{MeV} /$ nucleon. The calculated result by AMD with cluster correlations is compared to the FOPI experimental data [2].

\section{Clusters at $50 \mathrm{MeV} /$ nucleon}

Emission of light particles, in systems of various neutron-proton asymmetries, is expected to be a probe of nuclear matter properties such as the density dependence of the symmetry energy and the neutron-proton effective mass difference (see Ref. [14] and references therein). It is evidently important to have a good theoretical understanding of emission of clusters.

The left part of Fig. 3 shows the calculated distributions of the emitted light particles as functions of the energy per nucleon $\varepsilon=E / A$ in the centerof-mass system in the central collisions of neutron-rich nuclei ${ }^{124} \mathrm{Sn}+{ }^{124} \mathrm{Sn}$ at $50 \mathrm{MeV} /$ nucleon. The distributions are weighted by the mass number of the particles. It is clearly seen that a large part of nucleons are bound in $\alpha$ particles in the low energy part. The neutron-proton ratio is $(N / Z)_{\text {gas }} \approx 2$ for the sum of these particles $(A \leq 4)$. This is significantly larger than the ratio $(N / Z)_{\text {system }}=1.48$ for the total system, which is understood as the effect of neutron fractionation. Since the same numbers of neutrons and protons are bound in many $\alpha$ particles (and deuterons), only a small number of protons and ${ }^{3} \mathrm{He}$ are left. Consequently the free $n / p$ ratio and the $t /{ }^{3} \mathrm{He}$ ratio can be very large, $\left(N_{\text {gas }}-2 n_{\alpha}\right) /\left(Z_{\text {gas }}-2 n_{\alpha}\right) \gg(N / Z)_{\text {gas }}$. In the right part of Fig. 3, the free $n / p$ ratio is compared with the experimental values 

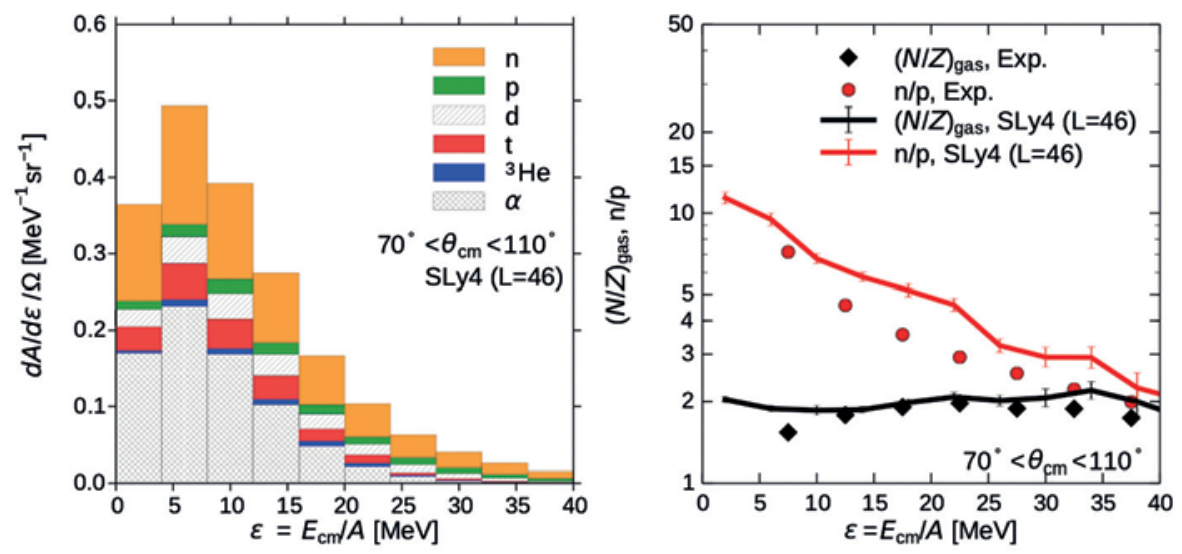

Figure 3: Left: The mass-weighted distributions of the energies $\varepsilon=E / A$ of light particles emitted to the angles $70^{\circ}<\theta<110^{\circ}$ in the center-of-mass system of ${ }^{124} \mathrm{Sn}+{ }^{124} \mathrm{Sn}$ central collisions at $50 \mathrm{MeV} /$ nucleon. Right: The spectral $n / p$ ratio of free neutrons and protons and the $(N / Z)_{\text {gas }}$ ratio of the sum of light particles.

from Ref. [14]. The $n / p$ ratio can be very large ( $\sim 10$ at low energies) due to the above-mentioned effect of the $\alpha$-particle formation. The exact value of the $n / p$ ratio is probably quite sensitive to the details of the emission of $\alpha$ particles. On the other hand, the $(N / Z)_{\text {gas }}$ ratio for the sum of these light particles takes a moderate value $(\sim 2)$ without a strong dependence on the energy $\varepsilon$. It seems that the present calculation with the Skyrme SLy4 interaction (corresponding to $L=46 \mathrm{MeV}$ ) is slightly overestimating $(N / Z)_{\text {gas }}$ compared to the data.

\section{Nuetron-proton dynamics at $300 \mathrm{MeV} /$ nucleon}

Heavy-ion collisions at several hundred $\mathrm{MeV} /$ nucleon provide us an opportunity to explore compressed nuclear matter. In the AMD calculation with clusters, the maximum density slightly higher than $2 \rho_{0}$ is reached at about $t=20 \mathrm{fm} / c$, in ${ }^{132} \mathrm{Sn}+{ }^{124} \mathrm{Sn}$ central collisions at $300 \mathrm{MeV} /$ nucleon. In such neutron rich systems, the neutron-proton ratio in the compressed part of the system is sensitive to the value of the symmetry energy at high densities [15]. The left part of Fig. 4 shows the neutron-proton density difference at $t=25$ $\mathrm{fm} / c$ defined by $\Delta_{n p}(r)=4 \pi r^{2}\left[(A / N) \rho_{n}(r)-(A / Z) \rho_{p}(r)\right]$ for the angleaveraged densities $\rho_{n}(r)$ and $\rho_{p}(r)$. The coefficients are defined by using $A$, $N$ and $Z$ of the reaction system so that we have $\int_{0}^{\infty} \Delta_{n p}(r) d r=0$. The results are shown by two lines for the effective interactions corresponding to 

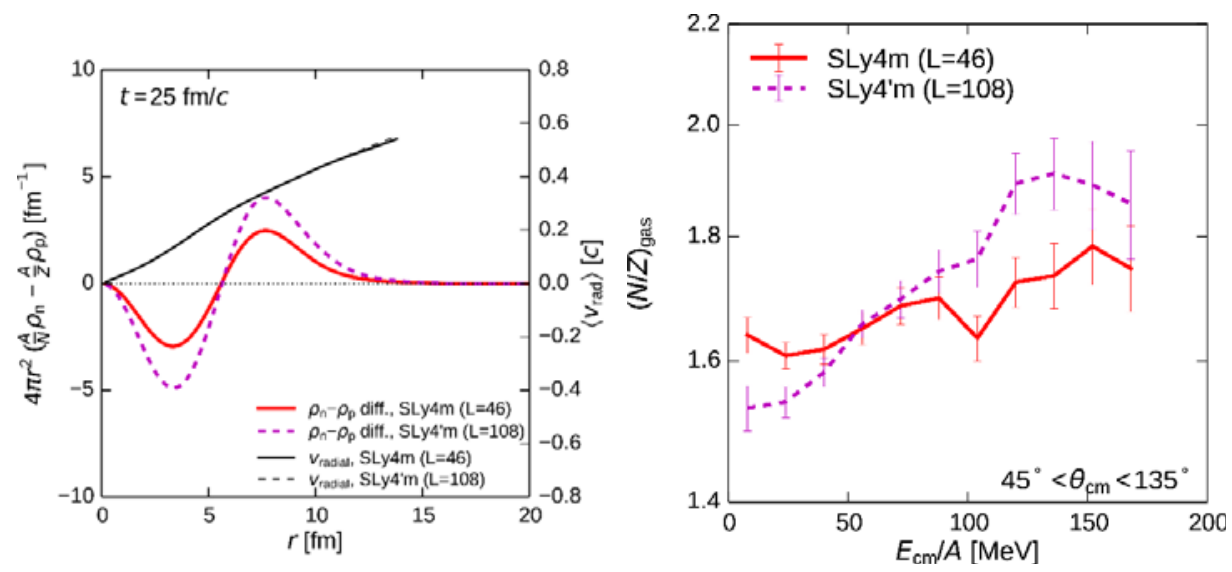

Figure 4: Left: The difference between neutron and protons densities defined as in the text as a function of the distance from the center of the system at the time $t=25 \mathrm{fm} / c$ in ${ }^{132} \mathrm{Sn}+{ }^{124} \mathrm{Sn}$ central collisions at $300 \mathrm{MeV} /$ nucleon. The collective radial velocity is also shown. Right: The $(N / Z)_{\text {gas }}$ ratio for the sum of emitted particles $1 \leq A \leq 4$ for the final state of the same reaction system.

$L=46 \mathrm{MeV}$ (solid) and $L=108 \mathrm{MeV}$ (dashed). As expected, the central high-density part becomes less neutron rich than the exterior low-density part, and the effect is stronger for the stiffer symmetry energy.

The AMD calculations here were performed with the free nucleonnucleon cross sections, with a parameter set for cluster correlations different from Sec. 2. For the stiffer symmetry energy, the density dependent term of the Skyrme SLy4 interaction $v_{\rho}^{(L=46)}=\frac{1}{6} t_{3}\left(1+x_{3} P_{\sigma}\right) \rho\left(\mathbf{r}_{1}\right)^{\alpha} \delta\left(\mathbf{r}_{1}-\mathbf{r}_{2}\right)$ was replaced by $v_{\rho}^{(L=108)}=\frac{1}{6} t_{3}\left(1+x_{3}^{\prime} P_{\sigma}\right) \delta\left(\mathbf{r}_{1}-\mathbf{r}_{2}\right) \rho\left(\mathbf{r}_{1}\right)^{\alpha}+\frac{1}{6} t_{3}\left(x_{3}-x_{3}^{\prime}\right) \rho_{0}^{\alpha} P_{\sigma} \delta\left(\mathbf{r}_{1}-\right.$ $\mathbf{r}_{2}$ ) with $x_{3}^{\prime}=-0.5$. The momentum dependence of the energy functional was modified for high energy collisions in a similar way to Ref. [16].

An important question is, of course, how this effect at an early stage can be observed in experiments. The charged pion ratio can be a good probe [15]. We here explore another complementary possibility of using spectra of nucleons and clusters. A hint is found in the left part of Fig. 4 in which the radial expansion velocity is also shown. The almost linear dependence on the distance from the center suggests that the system is very simply expanding in this high energy collision. Therefore the exterior part, which is neutron rich, will contribute to the high velocity particles in the final state, while the neutron-poor interior part will be observed as low velocity particles. In fact, this very simple picture seems correct in the calculation as in the right part of Fig. 4 which shows the $(N / Z)_{\text {gas }}$ spectral ratio for 
the sum of light particles $1 \leq A \leq 4$. The relation between the two lines for the different density dependences of the symmetry energy in the $(N / Z)_{\text {gas }}$ spectral ratio is similar to $\Delta_{n p}(r)$ in the left part.

It should be noted that in the literature the $n / p$ spectral ratio has been found to be insensitive to the density dependence of the symmetry energy, while it is sensitive to the neutron-proton effective mass difference [17], based on the stochastic mean field (SMF) calculation without cluster correlations. On the other hand, the present AMD calculation takes into account the cluster correlations. In fact, when we turn off the cluster correlations in AMD, we find the sensitivity to the symmetry energy is lost in the $(N / Z)_{\text {gas }}$ spectral ratio. This suggests that the expansion dynamics is largely influenced by cluster correlations, which is understandable because the number of particles (namely clusters and nucleons) is smaller in the calculation with cluster correlations than without them. The interaction between particles ceases at an earlier time with cluster correlations, while the interaction between nucleons continues longer in the calculations without clusters so that the final neutron and proton spectra are modified from those at early times. This effect is similar to what we found in a different context in the comparison of AMD and SMF [18].

\section{Summary}

Clusters are important in heavy-ion collisions, not only simply because they are emitted, but also because formation and existence of light clusters influence very much the global reaction dynamics and the bulk nuclear matter properties. AMD has been extended to include cluster correlations in the final states of two-nucleon collisions. The binding of several clusters to form a nucleus should also be considered. The consistent reproduction of basic character of multifragmentation is much more satisfactory than before. Some observables, such as the $n / p$ spectral ratio, are sensitive to the $\alpha$-particle formation. If cluster correlation is strong, the expansion is simple in collsions at $300 \mathrm{MeV} /$ nucleon so that the high-density effect of the symmetry energy is reflected almost directly in the $(N / Z)_{\text {gas }}$ spectral ratio.

\section{Acknowledgments}

This work was supported by JSPS KAKENHI Grant Numbers 21540253 and 24105008. The numerical calculations were partly carried out on SR16000 at YITP in Kyoto University. 


\section{References}

[1] S. Hudan et al., Phys. Rev. C 67 (2003) 064613.

[2] W. Reisdorf et al., Nucl. Phys. A 848 (2010) 366.

[3] B. Borderie et al., Eur. Phys. J. A 6 (1999) 197.

[4] K. Sumiyoshi and G. Röpke, Phys. Rev. C 77 (2008) 055804.

[5] L. Qin et al.,, Phys. Rev. Lett. 108 (2012) 172701.

[6] M. Hempel, K. Hagel, J. Natowitz, G. Röpke and S. Typel, Phys. Rev. C 91 (2015) 045805.

[7] G. Röpke, Nucl. Phys. A 867 (2011) 66.

[8] P. Danielewicz and G.F. Beartsch, Nucl. Phys. A 533 (1991) 712.

[9] D.D.S. Coupland, W.G. Lynch, M.B. Tsang, P. Danielewicz and Y. Zhang, Phys. Rev. C 84 (2011) 054603.

[10] A. Ono, Journal of Physics: Conference Series 420 (2013) 012103.

[11] A. Ono, H. Horiuchi, T. Maruyama and A. Ohnishi, Prog. Theor. Phys. 87 (1992) 1185.

[12] Y. Kanada-En'yo, M. Kimura and A. Ono, Prog. Theor. Exp. Phys. 2012 (2012) 01A202.

[13] K. Hagel et al., Phys. Rev. C 50 (1994) 2017.

[14] D.D.S. Coupland et al., arXiv:1006.4546 [nucl-ex] (2014).

[15] B. A. Li, Phys. Rev. Lett. 88 (2002) 192701.

[16] C. Gale, G. Bertsch, and S. Das Gupta, Phys. Rev. C 35 (1987) 1666.

[17] V. Giordano, M. Colonna, M. Di Toro, V. Greco and J. Rizzo, Phys. rev. C 81 (2010) 044611.

[18] M. Colonna, A. Ono and J. Rizzo, Phys. Rev. C 82 (2010) 054613. 